\title{
Cardiac sarcoidosis: from diagnosis to treatment
}

\author{
Kristyna Kysperska, Petr Kuchynka, Tomas Palecek
}

Sarcoidosis is a systemic granulomatous disease of unknown cause. Its clinical presentations are heterogeneous and virtually any organ system can be affected, most commonly lungs. The manifestations of cardiac sarcoidosis (CS) are heterogenous depending on the extent and location of the disease and range from asymptomatic forms to lifethreatening arrhythmias as well as to progressive heart failure. Cardiac involvement is associated with a worse prognosis. The diagnosis of CS is often challenging and requires a multimodality approach based on current international recommendations. Pharmacological treatment of CS is based on administration of anti-inflammatory therapy (mainly corticosteroids), which is often combined with heart failure medication and/or antiarrhythmics. Nonpharmacological therapeutic approaches in CS cover pacemaker or defibrillator implantation, catheter ablations and heart transplantation. This review aims to summarize the current understanding of CS including its epidemiology, etiopathogenesis, clinical presentations, diagnostic approaches, and therapeutic possibilities.

Key words: granulomas, FDG-PET/CT, conduction disorders, ventricular arrhythmias, heart failure

Received: July 8, 2021; Revised: September 22, 2021; Accepted: September 30, 2021; Available online: October 14, 2021 https://doi.org/10.5507/bp.2021.057

(c) 2021 The Authors; https://creativecommons.org/licenses/by/4.0/

$2^{\text {nd }}$ Department of Medicine - Department of Cardiovascular Medicine, First Faculty of Medicine, Charles University in Prague and General University Hospital in Prague, Prague, Czech Republic

Corresponding author: Tomas Palecek, e-mail: tomas.palecek@lf1.cuni.cz

\section{INTRODUCTION}

Sarcoidosis is a systemic granulomatous inflammatory disease of unknown aetiology. It is characterized histologically by the presence of non-caseating granulomas. It can involve any organ system, most commonly lungs, heart, liver, skin, gastrointestinal and neurologic system ${ }^{1}$. A wide range of clinical manifestations is therefore possible including asymptomatic patients to victims of sudden death on the other side. Importantly, cardiac sarcoidosis has a significant impact on prognosis of the patient. Increased understanding and awareness of this disease are thus of utmost importance. It is necessary to establish diagnostic and treatment standards based on randomised studies, screen asymptomatic patients and monitor the response to treatment.

\section{EPIDEMIOLOGY}

The incidence and prevalence of sarcoidosis are highly variable and depend on geographical region, gender, ethnicity, and age. The average prevalence is estimated to be 4.7-64 in 100000 and more common in females than males ${ }^{2,3}$. Sarcoidosis is usually diagnosed between 40 to 55 years of age. However, the onset of the disease seems to have a biphasic pattern with one peak at the third decade of life and the other one in the sixth decade ${ }^{3}$. An US population-based study reported annual prevalence of 5.9 in 100000 men and 6.3 in 100000 women, respectively ${ }^{4}$. The incidence is highest in Northern Europe (11-24 cases per 100 000/year) (ref..$\left.^{5}\right)$ and among African
Americans (17-71 cases per 100 000/year) (ref. $\left.{ }^{6}\right)$; the lowest incidence is reported in Asian countries ( 1 case per $100000 /$ year) $\left(\right.$ ref. $\left.^{7}\right)$.

\section{AETIOLOGY, GENETIC PREDISPOSITION, AND ENVIRONMENTAL FACTORS}

The exact aetiology of sarcoidosis is still unknown. The onset of the disease and its course are influenced by many factors. Genetic predisposition seems to play an important role. Having a family member with the disease is associated with a 2 to 4 -fold increased risk of developing sarcoidosis ${ }^{8}$. The risk is particularly high in first degree relatives. The relative risk in family members has been reported as 8.1 in the Japan study ${ }^{9}$ and 18.0 in the American study ${ }^{10}$.

Human leukocyte antigens (HLA) class II are presumed to be associated with disease susceptibility, its course as well as prognosis. Both Japanese ${ }^{11}$ and European studies $^{12}$ reported higher frequency of several HLA alleles such as DRB1 in patients with sarcoidosis. The HLADRB $1{ }^{*} 03$ allele has been shown to be significantly associated with Löfgren's syndrome, acute and self-limiting form of sarcoidosis. On the other hand, HLA-DRB $1 * 14$ and $-\mathrm{DRB} 1 * 15$ alleles are frequently found in patients with a chronic course of the disease ${ }^{13}$. Furthermore, mutations in other genes relate to immune response such as through chemokine receptors that likely play a role in aetiology of sarcoidosis.

Environmental and lifestyle factors also have an impact on the course of the disease. Mouldy environment, 
exposure to insecticides and agricultural employment are discussed in association with higher risk of developing sarcoidosis. The risk seems to be increased in other occupations including iron foundry work and firefighters, possibly due to exposure to silica dust. Importantly, the prevalence of sarcoidosis is higher in densely populated areas $^{3}$.

Body weight also seems to influence the risk of sarcoidosis development. In a Black women's health study, obesity was associated with a $40 \%$ increased risk of sarcoidosis $^{14}$. Also, in a Nurses' health American study the authors demonstrated a $70 \%$ increased risk of disease in obese individuals ${ }^{15}$. These studies also reported a relationship between high body mass index at 18 years of age and subsequent increase in incidence of sarcoidosis in adulthood.

Hormones may play an important role in the development of sarcoidosis as suggested by gender differences and a biphasic age pattern in the disease manifestation. Higher endogenous oestrogen levels, later age at first delivery and recent delivery seem to be protective factors associated with lower risk of sarcoidosis ${ }^{3}$.

\section{PATHOGENESIS}

Sarcoidosis is characterized by epitheloid, non-caseating, non-necrotizing granulomas with varying degrees of lymphocytic inflammation. The granulomas are compact, centrally organized areas of accumulated macrophages, which originate from activated $\mathrm{T}$ helper and epitheloid cells, surrounded by lymphocytes. Of note, granulomas are not specific for sarcoidosis, they can occur in multiple other diseases like cancer, infectious granulomatous diseases, chronic beryllium disease and inflammatory response to inorganic foreign material.

Generally, IV type reactions of hypersensitivity are present. The exposure to unknown specific antigen initiates an innate immune response in genetically predisposed individuals. Antigen recognition by $\mathrm{T}$ cells starts the recruitment of more specific T-clones and triggers proinflammatory cytokine production (interferon gamma, tumour necrosis factor alpha, interleukins $12,15,16$, 17, 18 and granulocytes macrophage colony stimulating factor) which activates B cells and the development of humoral response. Lymphocytes located in affected tissue consist of CD4+T helper cells and differentiate into TH1, TH17 effector cells. Proinflammatory cytokine production is upregulated due to unknown factors. On the other hand, the number of $\mathrm{T}$ regulatory cells and natural killer $\mathrm{T}$ cells is decreased, and their function is impaired. Hypergammaglobulinemia is also present. The above described dysregulated and aggravated immune response finally leads to formation of granulomas with the aim to isolate undegradable antigens. Amplification of $\mathrm{T}$ immune response to local tissue antigens also leads to increased accumulation of serum amyloid $\mathrm{A}$ in resident macrophages and granulomas. The persistence and progression of granulomas into chronic inflammation with subsequent development of fibrotic tissue is moderated by so far unknown factors ${ }^{3}$.

Activated $\mathrm{T}$ cells always express identical nucleotide sequence of variable gene segment of TCR, with higher frequency in patients with HLA-D3. Therefore, only one or several antigens are assumed to trigger sarcoidosis. In 1940s, a specific reagent prepared from the spleen of affected patients was used as a diagnostic test ${ }^{16}$. A granulomatous skin reaction was induced in $70-80 \%$ of untreated patients with sarcoidosis but not in healthy people, probably due to the presence of disease-triggering antigen. Currently this reagent is forbidden because of risk of transferring infection ${ }^{17}$.

Vimentin is assumed to be a potential autoantigen not only in rheumatoid arthritis and systemic lupus erythematosus but also in sarcoidosis. It is an intermediate filament protein secreted by activated macrophages in granulomas. Studies have documented that V $\alpha 2.3 / \mathrm{V} \beta 22$ T-cell receptor-expressing $\mathrm{CD}^{+}{ }^{+} \mathrm{T}$-cells accumulate in the lungs of HLA-DR3 patients ${ }^{3}$.

Microorganisms are also assumed to play a role in pathogenesis of sarcoidosis. A seasonal variation in the incidence of sarcoidosis has been reported. The transfer of sarcoidosis from donor to recipient during bone marrow transplantation was also documented ${ }^{3}$. The non-degradable remnants of microorganisms like Mycobacterium tuberculosis and Cutibacterium acnes or molecular mimicry may be a target of adaptive immune response and triggered T helper cells immune response. Such intracellular pathogens may lead to onset of granuloma formation during latent infection. Indeed, the development of granulomas was observed in people with an allergic disposition to Mycobacterium tuberculosis ${ }^{4}$. The levels of nucleic acids of Mycobacterium tuberculosis as well as of Cutibacterium acnes were detected 10-20 times more frequently in tissue samples taken from patients with sarcoidosis than in healthy individuals ${ }^{18}$. Furthermore, antibodies against Mycobacterium tuberculosis remnants were found in biopsy tissue of $39 \%$ patients with sarcoidosis ${ }^{19}$. On the other hand, until now there has been no evidence of any living microorganism in granulomatous tissue.

\section{PREVALENCE OF CARDIAC SARCOIDOSIS}

Cardiac involvement in sarcoidosis is associated with poor prognosis. The heart may be affected as a part of systemic involvement or as an isolated cardiac disease. Whether the isolated cardiac sarcoidosis is prognostically different than the systemic form of the disease with cardiac involvement is currently not clear.

According to autopsy studies, cardiac involvement may be found in about $25 \%$ of patients with systemic disease ${ }^{20,21}$. However, only $5 \%$ of patients with systemic sarcoidosis are described to have clinically manifested cardiac sarcoidosis. The results of studies using advanced cardiac imaging suggest $30 \%$ of all affected patients express cardiac involvement, regardless of symptoms ${ }^{22}$.

The prevalence of isolated cardiac involvement has 
been reported between $27-54 \%$, but this high number may be due to low sensitivity of the diagnostics of extracardiac involvement ${ }^{2}$. Importantly, the prevalence of sarcoidosis together with giant-cell myocarditis in patients $<55$ years with initially unexplained high degree AV blocks is up to $25 \%$ (ref. $^{23}$ ). Moreover, arrhythmogenic inflammatory cardiomyopathy as diagnosed by positive FDG PET-CT scan, i.e. presumably predominantly cardiac sarcoidosis, was documented in nearly $50 \%$ of patients with unexplained cardiomyopathy and ventricular arrhythmias ${ }^{24}$ and in 4,4 $-12,5 \%$ in patients with non-ischemic dilated cardiomyopathy ${ }^{22,25}$. The study based on the United Network for Organ Sharing Database showed that cardiac sarcoidosis was the most misclassified cause of dilated cardiomyopathy in patients undergoing heart transplantation ${ }^{26}$.

\section{CLINICAL PRESENTATION OF CARDIAC SARCOIDOSIS}

The clinical manifestations depend on location and extension of heart involvement. In fact, the disease may affect almost any part of the heart including ventricular and atrial myocardium, conduction system and pericardium. Cardiac abnormalities can be the first and sometimes the only manifestation of sarcoidosis. Sarcoidosis of the heart may remain asymptomatic or present as dilated or restrictive cardiomyopathy associated with progressive heart failure, conduction system disorders, especially highgrade atrioventricular blocks, and ventricular arrhythmias. Heart failure, whether with low or preserved LV EF, represents the most important cause of death in cardiac sarcoidosis, accounting for $25 \%$ of mortality in patients with cardiac involvement. Valvular and pericardial involvement is relatively rare. According to some studies, sudden cardiac death accounts for $13-25 \%$ of sarcoidosis-related deaths and may be the first manifestation of the disease ${ }^{4}$.

Conduction abnormalities. Sarcoidosis can affect any part of the conduction system. Atrioventricular blocks are a major complication and may be found as an initial manifestation of the disease in $23-77 \%$ patients ${ }^{4}$. Their cause is connected to the affection of the basal interventricular septum and atrioventricular node by granulomatous infection and subsequent fibrosis leading to the thinning of this myocardial region. Importantly, the onset and degree of atrioventricular blocks is usually not associated with the development of left ventricular systolic dysfunction. Due to their prevalence, all patients younger than 60 years with unexplained high degree A-V block should be screened for cardiac sarcoidosis by CMR or FDG-PET as stated in Heart Rhythm Society Expert Consensus Statement on arrhythmias associated with cardiac sarcoidosis algorithm from 2014 (ref. ${ }^{27}$ ). The presence of AV block may be asymptomatic or may manifest as palpitations, dizziness, and syncope or even as sudden death in most advanced cases.

Ventricular arrhythmias. Inflammation and fibrosis of the myocardium may cause ventricular arrhythmias covering asymptomatic or symptomatic premature ventricular beats as well as ventricular tachycardias and ventricular fibrillation. Malign ventricular tachyarrhythmias often develop in patients with at least mildly decreased LV systolic function; however, they can be present also in subjects with preserved LV ejection fraction. Right ventricular involvement may be associated with ventricular arrhythmias mimicking clinical scenario of arrhythmogenic right ventricular cardiomyopathy. The origin of ventricular arrhythmias is usually connected to re-entrant pathways caused by myocardial scaring; nevertheless, triggered activity or abnormal automaticity may also be present. In general, ventricular arrhythmias are observed in about $23-36 \%$ patients with newly diagnosed cardiac sarcoidosis ${ }^{4}$. Interestingly, so far, no relationship has been demonstrated between the activity of the disease as assessed by imaging techniques and genesis of ventricular $\operatorname{arrhythmias}^{28}$.

Heart failure. The signs and symptoms of heart failure reflect fluid retention and low cardiac output, respectively, and consist of progressive dyspnoea, cough, peripheral oedema, malaise, and dizziness. Heart failure in patients with sarcoidosis results from myocardial involvement, which typically manifests as a dilated cardiomyopathy with various degrees of reduced ejection fraction and impaired filling. Restrictive cardiomyopathy is still cited as another possible manifestation of cardiac sarcoidosis; however, it is rarely diagnosed in routine clinical practice $^{22}$.

\section{DIAGNOSIS OF CARDIAC SARCOIDOSIS}

Timely diagnosis of cardiac sarcoidosis is essential because early initiation of therapy improves the clinical status of the patient and has a significant impact on further progression of the disease. The diagnosis of cardiac sarcoidosis is typically done in one of two clinical scenarios: during screening of patients with already diagnosed extracardiac (usually pulmonary) sarcoidosis, or in subjects suffering from various cardiac symptoms of unknown cause described above. However, some patients are diagnosed only at autopsy or based on histological examination of explanted heart at a time of cardiac transplantation.

\section{ECG}

Abnormal ECG findings are present in up to $90 \%$ of patients with cardiac sarcoidosis. As already described, typical abnormalities are represented by conduction system disorders and ventricular arrhythmias; however, other ECG changes like atrial arrhythmias, pathological $\mathrm{Q}$ waves and repolarization abnormalities may be also present ${ }^{4}$. Indeed, any ECG abnormality may be seen in cardiac sarcoidosis. Right bundle branch block is an often finding, which is seen in about $12-66 \%$ of patients. Although it is not specific, it shall be regarded as a very suspicious marker of myocardial involvement in individuals with dilated cardiomyopathy and extracardiac sarcoidosis $^{29}$. 


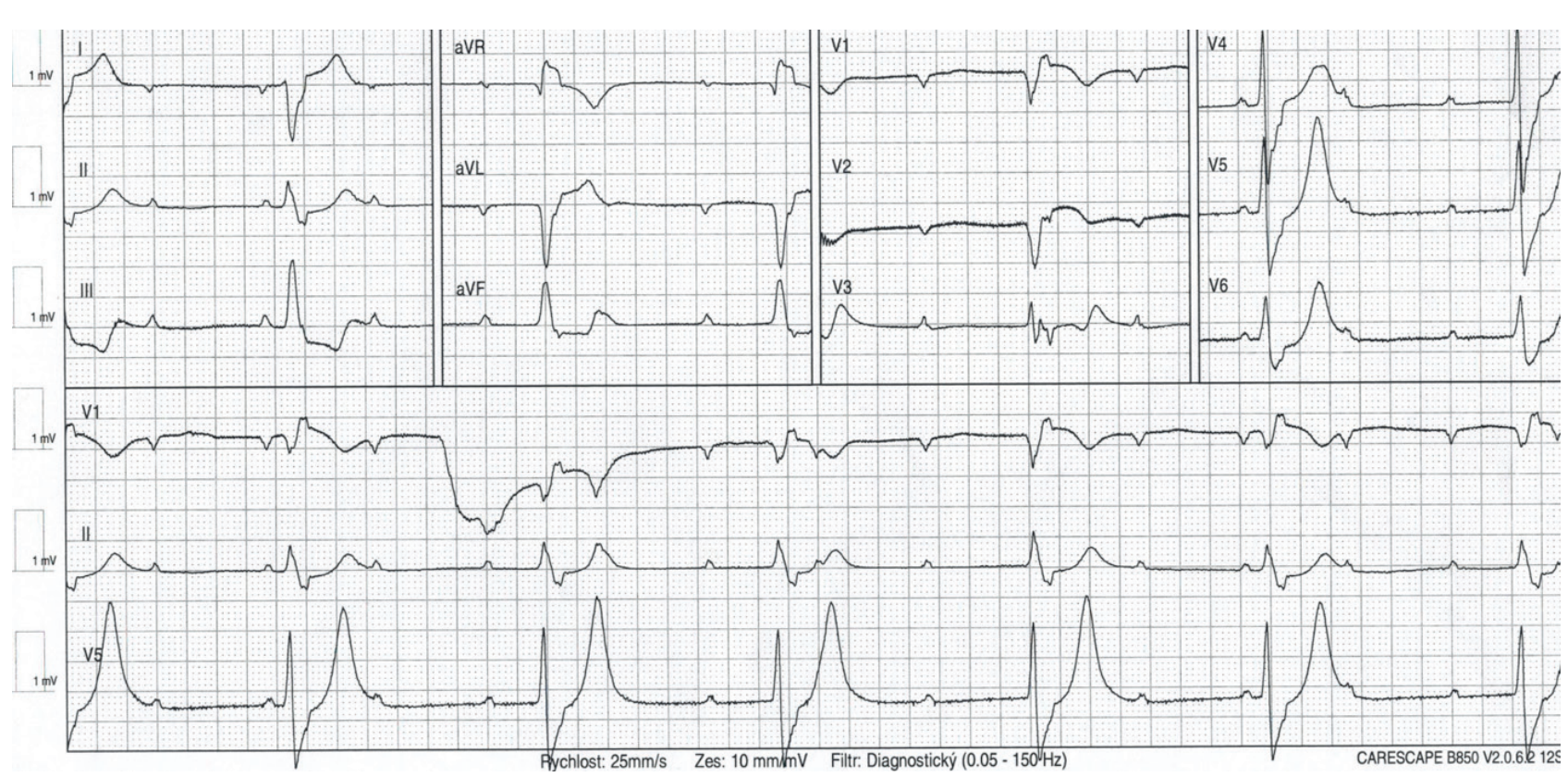

Fig. 1. 12-lead ECG showing third-degree atrioventricular (AV) block in a patient with cardiac sarcoidosis.

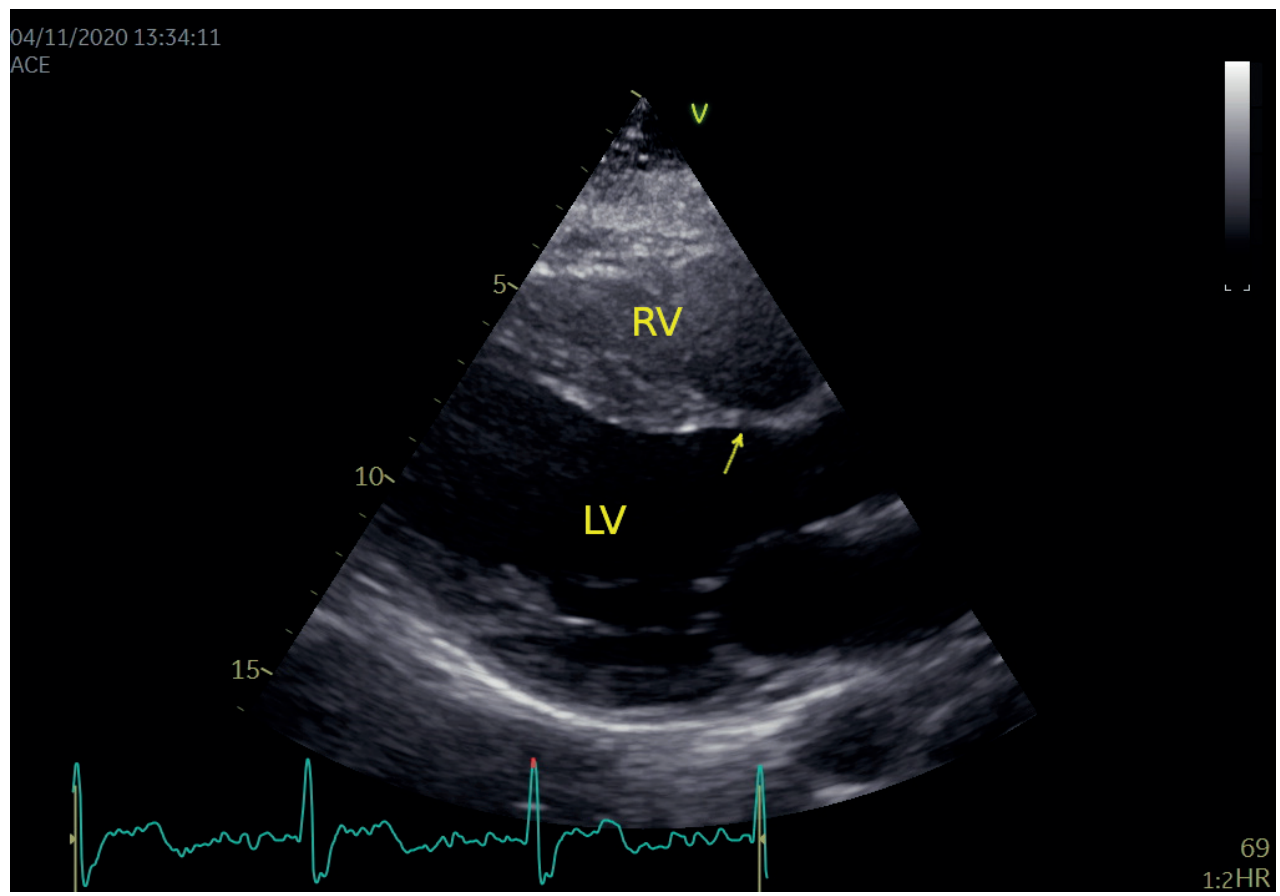

Fig. 2. Transthoracic echocardiography in parasternal long-axis view demonstrating localized thinning of the basal interventricular septum (arrow) in a patient with cardiac sarcoidosis (LV, left ventricle; RV, right ventricle).

AV block represents the most common initial manifestation of cardiac sarcoidosis (Fig. 1). Importantly, cardiac sarcoidosis may be found in up to $25 \%$ of young and middle-aged patients with initially unexplained high-degree AV block as shown in the landmark study published by Kandolin et al. ${ }^{23}$. Malignant ventricular arrhythmias are observed in $23-36 \%$ of affected individuals. In some cases, intractable arrhythmic storm is caused by several arrhythmogenic foci located in scattered myocardium. The occurrence of ventricular arrhythmias is often connected with the fibrotic thinning of the basal interventricular sep- tum or left or right ventricular free walls. Nevertheless, the are not associated with global left ventricular systolic dysfunction ${ }^{4}$.

\section{Echocardiography}

Due to its wide availability, safety and relatively low cost, echocardiography still represents the first line imaging method for diagnosing cardiac sarcoidosis. Moreover, it is the method of choice for routine monitoring of patients with already diagnosed cardiac sarcoidosis. However, its sensitivity in initial stages of cardiac 
sarcoidosis seems to be very low. Several important echocardiographic features of cardiac sarcoidosis have been reported. Regional thinning of the basal interventricular septum associated with wall motion abnormality in this region is highly sensitive and rather specific finding of cardiac sarcoidosis (Fig. 2). It is defined as wall thickness less then $4 \mathrm{~mm}$ measured at $10 \mathrm{~mm}$ far from aortic annulus in the parasternal long axis view. Another definition is based on the ratio of thickness of thinned and normal interventricular septum lower than 0.6 (ref. ${ }^{30}$ ). Of course, coronary disease as a cause of this wall motion abnormality must be ruled out. Furthermore, regional wall motion abnormalities of other walls may be found, and their uneven distribution is typical for cardiac sarcoidosis. They reflect the presence of scattered granulomas and non-ischemic fibrous scars in the myocardium. Apparent ventricular aneurysms are found in $8-10 \%$ of patients ${ }^{31}$.

Diffuse myocardial involvement leads to the picture of dilated cardiomyopathy characterized by the dilatation of the left ventricle and global impairment of its systolic function. The remodelling of the left ventricle together with mitral annular dilatation may lead to secondary mitral regurgitation; however, in some cases, mitral regurgitation is due to pronounced annular calcification associated with hypercalcemia that is often present in sarcoidosis. There is an increased risk of thrombus formation in poorly contracting dilated left ventricle.

Early subclinical myocardial dysfunction may be detected by strain echocardiography. Pizarro et al. have shown that global longitudinal strain less than $-14 \%$ is not only an independent predictor for the presence of cardiac sarcoidosis, but also for worse prognosis with respect to heart failure, new arrhythmias, and mortality, respectively ${ }^{32}$

Right ventricular involvement may be also present. In some patients, transient thickening of otherwise thin right ventricular free wall is found due to active granulomatous inflammation. However, regional wall motion abnormalities like local akinesia or aneurysm are detected more often and, in such cases, arrhythmogenic right ventricular cardiomyopathy must be taken into the account in differential diagnosis.

Pericardial effusion is found rarely, only in $2-8 \%$ of patients ${ }^{4}$.

\section{Cardiac Magnetic Resonance}

Cardiac magnetic resonance (CMR) has become one the most valuable imaging modalities in diagnostics of cardiac sarcoidosis. Using cine steady state free precession images, morphological and functional abnormalities of both ventricles may be detected similarly to echocardiography. Typical findings suggestive of cardiac sarcoidosis thus comprise thinning of the basal interventricular septum, regional wall motion abnormalities, or diffuse hypokinesis of dilated left ventricle. Akinesis or aneurysms of the RV free wall may mimic arrhythmogenic right ventricular cardiomyopathy.

The uniqueness of cardiac magnetic resonance lies in its ability to characterize tissue. Using T2 weighted black blood images, myocardial oedema as a sign of ac-

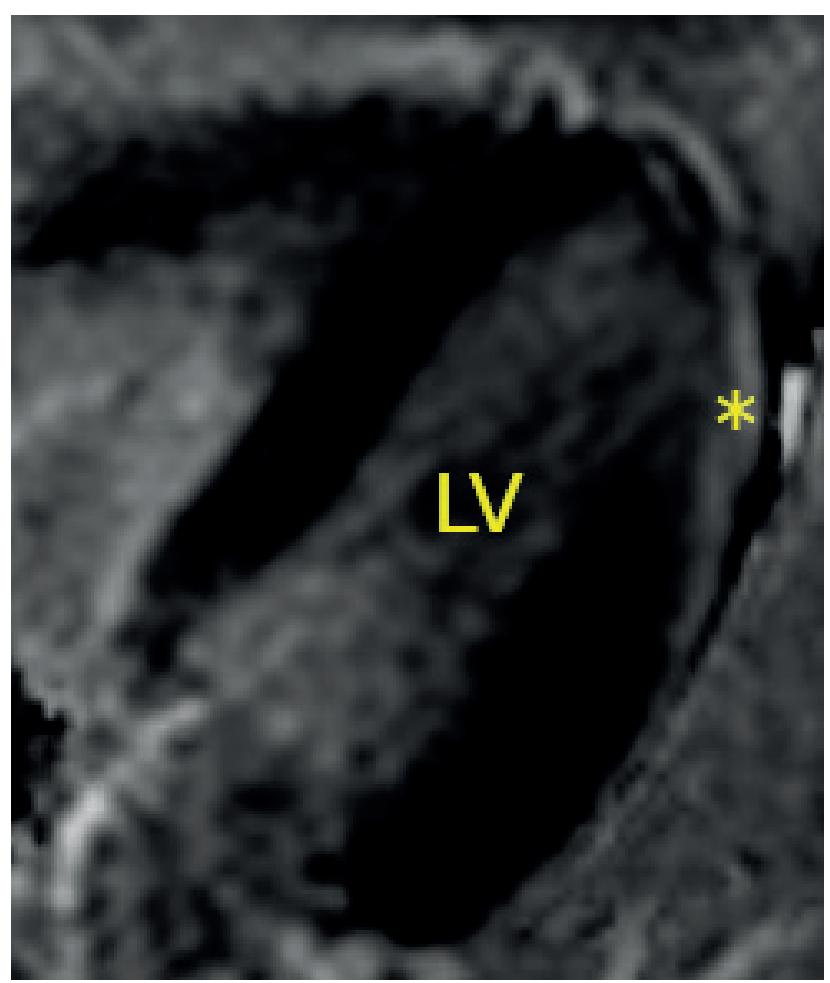

Fig. 3. Cardiac magnetic resonance imaging in four-chamber view showing subepicardial pattern of late gadolinium enhancement in the apical half of the left ventricular anterolateral wall (star) in a patient with cardiac sarcoidosis (LV, left ventricle).

tive myocardial inflammation may be visualised. However, the most important technique represents the assessment of late gadolinium enhancement (LGE). Generally, the presence of LGE reflects the expansion of extracellular space of the myocardium comprising acute necrosis or, in the chronic cases, replacement fibrosis or myocardial infiltration. In patients with cardiac sarcoidosis, LGE lesions are commonly found in thinned basal interventricular septum and in lateral wall (Fig. 3). Nevertheless, another localization of LGE is possible, typically in a multifocal, patchy distribution affecting mid-wall and subepicardium. Subendocardial LGE may also be found in some patients resembling coronary-pattern of LGE. In these cases, however, regional distribution of LGE usually does not correspond to the perfusion territory of the coronary artery. According to several studies, the sensitivity and specificity of LGE in detection of cardiac sarcoidosis reaches 78$100 \%$ and $76-92 \%$, respectively ${ }^{33}$. Typical LGE findings represent a part of Clinical diagnosis group Criteria for diagnosis cardiac sarcoidosis.

Cardiac magnetic resonance is useful not only in diagnostic process of cardiac sarcoidosis, but also in the assessment of prognosis of affected subjects and has the potential to monitor the effect of immunosuppressive therapy, especially when novel techniques for tissue characterization like T1 and T2 mapping are used. The metaanalysis of 10 studies with 760 patients demonstrated that the presence of LGE is associated with 1.7 times higher risk for all cause-mortality, in particular due to arrhythmic events $^{34}$. 


\section{Gallium scintigraphy}

Gallium scintigraphy uses radionuclide gallium-67 citrate to detect myocardial or pericardial inflammation or heart tumors. Since 1970s it has been used to detect active sarcoid lesions and to assess their response to antiinflammatory therapy. Gallium scintigraphy has relatively high specificity (about $80-100 \%$ ); however, its sensitivity is quite low, maximally $36 \%$. Its spatial resolution compared with FDG-PET is also low, especially for detecting extrapulmonary sarcoidosis ${ }^{4}$. Therefore, the use of gallium-67 citrate SPECT to diagnose cardiac sarcoidosis is currently limited to centers which do not have access to FDG-PET/ CT examination ${ }^{4}$.

\section{FDG-PET/CT}

This examination currently represents the gold non-invasive standard for monitoring of the immunosuppressive treatment of cardiac as well as extracardiac sarcoidosis. According to current recommendations for the diagnosis of cardiac sarcoidosis, PET-CT is indicated in the situation when CMR is unavailable or contraindicated, CMR examination result is unequivocal or negative and there is high clinical suspicion of cardiac sarcoidosis ${ }^{35}$. FDGPET/CT has an irreplaceable diagnostic value for detecting areas of active inflammation as granulomas express increased metabolic activity that results in higher uptake of fluorodeoxyglucose by immune cells in the affected myocardium. Adequate dietary preparation ("high fat and low carbohydrate" diet) before the examination is necessary to decrease blood glucose levels and increase free fatty acid levels to modify myocardial glucose uptake and highlight myocardial lesions from intact myocardium.

FDG PET-CT study is usually performed in combination with myocardial perfusion imaging. Using both techniques, diagnostic staging of cardiac sarcoidosis is possible to some extent. At initial phases, active inflammation on FDG-PET/CT is seen with no perfusion defects in the myocardium. With disease progression, myocardial perfusion defects reflecting myocardial scarring appear. In burn-out phase of the disease, no FDG activity, i.e. no inflammation, is detected by FDG-PET/CT while extensive areas of abnormal myocardial perfusion are present ${ }^{2}$. The meta-analysis of 7 studies comprising 891 patients showed $79-96 \%$ sensitivity and $68-86 \%$ specificity of FDGPET/CT for detection of cardiac sarcoidosis ${ }^{4,36}$. For more detailed staging of cardiac involvement, the combination of the information from FDG-PET/CT with myocardial perfusion study together with LGE findings on CMR may be used. According to recent retrospective study, the FDG-PET/CT sensitivity of detecting cardiac sarcoidosis increased from $82 \%$ to $94 \%$ when combined with CMR examination $^{37}$.

Importantly, FDG-PET/CT may be used not only for diagnostic reasons, but also for evaluating the prognosis of affected individuals and choosing the treatment with subsequent monitoring of its efficacy. The combined presence of higher FDG uptake and perfusion defects is associated with higher risk of ventricular tachyarrhythmias and death. Interestingly, the signs of inflammation in right ventricular wall have been shown to increase the risk of cardiac events fivefold, suggesting that right ventricle involvement could also serve as marker of disease severity ${ }^{38}$.

\section{Endomyocardial biopsy}

Endomyocardial biopsy has high specificity, but low sensitivity in diagnosing cardiac sarcoidosis. Moreover, its performance is associated with risk of life-threatening complications though very low in experienced centres. Therefore, extracardiac biopsy should be first performed in patients with other organ manifestations suspicious from sarcoidosis. According to current guidelines, the diagnosis of cardiac sarcoidosis can be established based on noninvasively obtained characteristic findings suggesting cardiac involvement and the verification of sarcoidosis in extracardiac localization, i.e. without the need for endomyocardial biopsy ${ }^{4}$.

Typical histological findings in sarcoidosis comprise non-caseating epithelioid granulomas, which consist of epithelioid cells, isolated multinucleation giant cells, macrophages, lymphocytes and plasma cells. Both myocardial necrosis and more than solitary eosinophils are typically absent. The degree of lymphocytic infiltration corresponds to stage of the disease. CD4+lymphocytes accumulate in granulomas and CD8+lymphocytes are found sporadically at the periphery of granulomas. During scarring stage, fibrotic tissue dominates with only small lymphocytic infiltration, while granulomas progressively disappear. In some lesions only giant cells or scar tissue can be found. Granulomas may affect the whole heart, but they typically present in focal manner. The most often affected place is the right side of the interventricular septum ${ }^{4}$.

When evaluating myocardial biopsy specimens, it is necessary to differentiate cardiac sarcoidosis from giant cells myocarditis. The latter is characterized by the presence of necrosis, eosinophilic infiltration and giant cells derived from macrophages and cardiomyocytes. Other pathologies like tuberculosis must be also ruled out by dedicated staining.

As already mentioned, untargeted endomyocardial biopsy has sensitivity only about $20-30 \%$, with high rate of false negative results. Periprocedural echocardiography may increase diagnostic yield by guiding the biopsy to myocardial regions with pathological findings seen on CMR or FDG-PET/CT. Importantly, periprocedural electroanatomic voltage mapping demonstrating local voltage abnormalities of the myocardium currently represents the most promising method for targeting of endomyocardial biopsy and thus increasing its sensitivity; however, its use is still limited ${ }^{39}$.

\section{Natriuretic peptides}

According to the study done on 172 patients with sarcoidosis, BNP is significantly increased in patients with cardiac sarcoidosis. The cut off value of $40 \mathrm{pg} / \mathrm{mL}$ for BNP and $125 \mathrm{pg} / \mathrm{mL}$ for NT-proBNP, respectively, can identify cardiac sarcoidosis with $85 \%$ sensitivity and $68 \%$ specificity. Higher values of these natriuretic peptides 
Table 1. Heart Rhythm Society 2014 Criteria for the Diagnosis of Cardiac Sarcoidosis (modified according to ref. ${ }^{41}$ ).

\begin{tabular}{ll}
\hline Histological diagnosis & $\begin{array}{l}\text { Endomyocardial biopsy specimen with non-caseating epitheloid granulomas and no alternative } \\
\text { cause identified (including negative organismal stains if applicable) }\end{array}$ \\
\hline Clinical diagnosis* & Histological diagnosis of extra-cardiac sarcoidosis \\
& and at least one of the following: \\
& - Steroid \pm immunosuppressant responsive cardiomyopathy or heart block \\
- Unexplained reduced LVEF (<40\%) & - Unexplained sustained (spontaneous or induced) VT \\
- Mobitz type II 2nd or 3rd degree atrioventricular block & - Patchy uptake on dedicated cardiac PET (in a pattern consistent with CS) and/or late \\
& gadolinium enhancement on CMR (in a pattern consistent with CS) and/or positive gallium \\
& uptake (in a pattern consistent with CS) \\
& and \\
Alternative causes for the cardiac manifestations excluded
\end{tabular}

* Clinical diagnosis is probable if above mentioned criteria are satisfied.

CMR, cardiac magnetic resonance; CS, cardiac sarcoidosis; LVEF, left ventricular ejection fraction; PET, positron emission tomography; VT, ventricular tachycardia.

also predict the development of heart failure ${ }^{40}$. Of course, elevated levels of natriuretic peptides cannot differentiate cardiac sarcoidosis from other cardiac pathologies. However, in recently published prospective study of 201 Polish patients, NT-proBNP level has been shown to be a factor associated with an increased risk of cardiac sarcoidosis, together with male gender, the presence of extrathoracic sarcoidosis, cardiac related symptoms, ECG abnormalities and contemporary radiological progression of pulmonary involvement ${ }^{41}$.

\section{Electrophysiological testing}

Ventricular tachycardias represent common manifestation of cardiac sarcoidosis and may be present in $23 \%$ of patients. Electrophysiological testing is used to assess the inducibility of ventricular arrhythmias to evaluate the risk of sudden cardiac death. The inducibility of ventricular tachycardia is not related to the activity of sarcoidosis, but it is associated with low LV EF and implies worse prognosis ${ }^{4}$.

In patients with spontaneous sustained ventricular arrhythmias, the risk of sudden death is increased 4.47 times. However, in patients with induced ventricular arrhythmias during electrophysiological testing the risk of sudden death is even 6.97 higher. In accordance, more than $50 \%$ of patients with spontaneous or inducible ventricular tachycardias get adequate ICD shock within 1 year after device implantation ${ }^{4}$. In study by Mehta et al. even $10 \%$ of patients with no symptoms of cardiac sarcoidosis who were diagnosed only by imaging findings had inducible ventricular arrhythmias and received ICD. In 5.5 years of follow-up, ventricular tachycardias appeared in $75 \%$ of those with initially inducible arrhythmia and only in $1.5 \%$ without it. Of note, average LV EF was $36.4 \%$ in patients with inducible arrhythmias, whereas $55.8 \%$ in subjects without them ${ }^{42}$.

\section{DIAGNOSTIC CRITERIA OF CARDIAC SARCOIDOSIS}

There are currently 2 major recommendations for diagnosis of cardiac sarcoidosis. According to the Heart Rhythm Society (HRS) consensus statement criteria, two diagnostic pathways of cardiac sarcoidosis are possible. The first one is based on direct histological prove of sarcoidosis in the sample of myocardial tissue; the other combines histological diagnosis of extracardiac sarcoidosis together with at least one of the clinical or imaging criteria for cardiac disease (Table 1). Therefore, histological finding of epithelioid granulomas in any organ is still necessary to diagnose sarcoidosis in general and isolated cardiac sarcoidosis can be diagnosed only by myocardial biopsy with all its known limitations and risks, namely low diagnostic yield ${ }^{43}$.

According to the Japanese Circulation Society guidelines, the histological diagnosis of cardiac sarcoidosis is done when non caseating epithelioid granulomas in myocardium are found through endomyocardial biopsy or cardiac surgery and other possible diagnoses were ruled out. Of note, these guidelines state, that the clinical diagnosis of cardiac sarcoidosis is currently possible even without performance of any biopsy. Clinically diagnosed cardiac sarcoidosis may be based on 1) the presence of epithelioid granulomas in organs other than heart together with the presence of clinical findings strongly suggestive of cardiac involvement, or 2) scintigraphic or FGD/PET-CT findings of abnormally higher tracer accumulation are present in heart and at least 3 other major criteria are fulfilled as well. The presence of strongly suggestive cardiac sarcoidosis is defined by two or more of the five major criteria, or one of the major criteria and two of three the minor criteria, listed in Table 2 (ref. ${ }^{4}$ ). 
Table 2. Japanese Circulation Society 2017 Criteria for the Diagnosis of Cardiac Sarcoidosis (modified according to ref.3).

Histological diagnosis Endomyocardial biopsy or surgical specimens demonstrate non-caseating epithelioid granulomas and other causes and local sarcoid reactions can be ruled out

Clinical diagnosis $\quad$ 1) Granulomas are found in organs other than the heart, and clinical findings are strongly suggestive of cardiac involvement; 2 ) or clinical findings are strongly suggestive of pulmonary or ophthalmic sarcoidosis and at least 2 of the 5 characteristic laboratory findings of sarcoidosis* are present and clinical findings strongly suggest of CS:

A. Two or more of the five major clinical criteria are satisfied

or

B. One of five of the major criteria and two or more of the three minor criteria are satisfied

Major criteria:

1. High-grade atrioventricular block (including complete atrioventricular block) or fatal ventricular arrhythmia

2. Basal thinning of the ventricular septum or abnormal ventricular wall anatomy (ventricular aneurysm, thinning of the middle or upper ventricular septum, regional ventricular wall thickening)

3. Left ventricular contractile dysfunction (ejection fraction less than $50 \%$ ) or focal ventricular wall asynergy

4. ${ }^{67}$ Ga-citrate scintigraphy or 18 F-FDG PET reveals abnormally high tracer accumulation in the heart

5. Gadolinium-enhanced CMR reveals delayed contrast enhancement of the myocardium

\section{Minor criteria:}

1. Abnormal ECG findings: non-sustained ventricular arrhythmias or multifocal or frequent premature ventricular contraction, bundle branch block, axis deviation, abnormal Q waves

2. Perfusion defects on myocardial perfusion scintigraphy

3. Endomyocardial biopsy: monocyte infiltration and moderate or severe myocardial interstitial fibrosis

Isolated cardiac sarcoidosis

1. No clinical findings characteristics of sarcoidosis are observed in any organs other than the heart

2. ${ }^{67}$ Ga- scintigraphy or 18 F-FDG PET reveals no abnormal tracer accumulation in any organs other than the heart.

3. A chest CT scan reveals no shadow along the lymphatic tracts in the lungs or no hilar and mediastinal lymphadenopathy (minor axis $>10 \mathrm{~mm}$ ) and

- Endomyocardial biopsy or surgical specimens demonstrate non-caseating epithelioid granulomas or

- ${ }^{67}$ Ga-scintigraphy or 18 F-FDG PET reveals abnormally trace accumulation are present in heart and at least three other major criteria are satisfied

- Endomyocardial biopsy or surgical specimens demonstrate non-caseating epithelioid granulomas or

- ${ }^{67}$ Ga-scintigraphy or 18 F-FDG PET reveals abnormally trace accumulation are present in heart and at least three other major criteria are satisfied Bilateral hilar lymphadenopathy; (2) High serum angiotensin-converting enzyme (ACE) activity or elevated serum lysozyme levels; (3) High serum soluble interleukin-2 receptor (sIL-2R) levels; (4) Significant tracer accumulation in 67Ga-citrate scintigraphy or 18F-FDG PET; (5) A high percentage of lymphocytes with a CD4/CD8 ratio of $>3.5$ in bronchoalveolar lavage fluid.

CMR, cardiac magnetic resonance; CS, cardiac sarcoidosis; CT, computer tomography; 18-FDG PET, fluorodeoxyglucose positron emission tomography.

\section{PROGNOSIS OF CARDIAC SARCOIDOSIS}

Cardiac involvement in sarcoidosis is generally associated with adverse prognosis. It has been shown to be a cause in about two-thirds of deaths in patients who died from sarcoidosis in Japan ${ }^{44}$. Other data from Japan demonstrated 1-year survival pf patients with cardiac sarcoidosis to be $85 \%$, and $60 \%$ and $44 \%$ in 5 - and 10 year survival, respectively ${ }^{45}$. More optimistic results have been reported recently by Finnish authors who described 1-year survival of $97.3 \%$ patients with cardiac sarcoidosis, with $90 \%$ and $83 \%$ surviving in subsequent 5 - and 10 -year follow-up, respectively ${ }^{46}$. The difference between results of these two studies may be related to the improvement of long-term prognosis of affected individuals caused by earlier initiation of corticosteroid therapy in the latter.

Several clinical, laboratory as well as imaging parameters have been shown to be associated with the prognosis of cardiac sarcoidosis. Not surprisingly, heart failure severity, LV EF together with troponin as well as natriuretic peptide levels are associated with worse prognosis ${ }^{22}$.

Of echocardiographic parameters other than LV EF, global longitudinal strain seems to be an independent prognostic factor. In a cohort of 100 Dutch patients, a cut-off value $-17 \%$ of global longitudinal strain predicted future development of LV systolic dysfunction, onset of heart failure, higher rate of hospitalizations, new dysrhythmias, and mortality of affected patients ${ }^{47}$. 
As in other myocardial diseases, the presence of replacement fibrosis, i.e. scar, demonstrated by CMR-LGE, has been shown to have a prognostic impact. Especially the presence of LGE in basal interventricular septum appears to be associated with worse prognosis, probably due to the location of cardiac conduction system in this region.

Cardiac FDG/PET-CT examination also provides prognostic information. Blankstein et al. showed, that the presence of perfusion defect together with increased FDG uptake should be regarded as a significant predictor of sustained ventricular tachycardias and death ${ }^{48}$. The prognostic importance of focal right ventricular FDG uptake was also demonstrated in this study.

\section{THERAPY OF SARCOIDOSIS - GENERAL PRINCIPLES}

The decision to initiate treatment of sarcoidosis is difficult because of rather unpredictable course of the disease. In many patients, the "watch and wait strategy" is applied and treatment delayed allowing spontaneous remission, that occurs in $50 \%$ of patients ${ }^{49}$. This approach requires careful and regular check-ups with monitoring of organ function over time. The indications for starting antiinflammatory therapy include progressive impairment of pulmonary function, major radiographic progression, ophthalmological manifestation, diabetes insipidus, lupus pernio, obstructive lymph nodes, renal or liver abnormalities, splenomegaly with thrombocytopenia, hypercalcemia, neurosarcoidosis and, importantly, any kind of cardiac involvement ${ }^{50}$.

Corticosteroids are currently the first line of therapy ${ }^{4}$. However, the prospective randomised clinical trials with systemic corticosteroid therapy have been conducted mainly in patients with pulmonary sarcoidosis an only limited reports have described their effects for extrapulmonary manifestations.

Other immunosuppressants are used as a second therapeutic line. They play an important role in treatment of corticosteroid-resistant disease, they can minimize the adverse effect of steroids by reducing their dose and shorten the duration of steroid therapy. Several reports of successful treatment with only non-steroid immunosuppressants have been published, but the combination with steroids is still dominantly used ${ }^{49}$. Methotrexate represents the most used steroid sparing agent. Its effect has been documented in skin, ocular, and neuromuscular sarcoidotic lesions as well as in chronic lesions of lung and heart. Azathioprine, another antimetabolite, has similar efficacy and significant steroid sparing potency as methotrexate; however, its use is associated with more infectious complications. Mycophenolate mofetil is a reversible inhibitor of inosine monophosphate dehydrogenase, that prevents proliferation of $\mathrm{T}$ and $\mathrm{B}$ lymphocytes and releases proinflammatory mediators in the granulomas. It can be used as an immunosuppressant after organ transplantation as well as in the treatment of inflammatory diseases. Its advantage lies in the absence of lung, hepatic and renal toxicity. Cyclophosphamide is usually indicated in severe cases when other therapeutic agents have failed. Leflunomide represents another alternative immunosuppressant with effect on pulmonary as well as extrapulmonary disease. It is often used as the third line of treatment in patients with progressive disease, who cannot tolerate other medication due to its toxic effects.

The principles of targeted treatment of sarcoidosis are based on the current knowledge of its immunopathogenesis. Tumor necrosis factor (TNF) represents a base mediator in recalcitrant sarcoidosis. It has a significant effect on granuloma formation as well as on progression of sarcoidosis. Both thalidomide and lenalidomide are immunomodulatory agents that inhibit production of TNF, and their effect was documented mainly in cutaneous form of the disease. Infliximab and its humanized form adalimumab belong to monoclonal antiTNF-alpha antibodies which are used in cases of refractory sarcoidosis, predominantly extrapulmonary. Anticytokine monoclonal antibodies represent highly specific approach to affect the inflammatory process; however, the possibility of severe infectious or autoimmune complications limits their use $\mathrm{e}^{50}$.

Bisphosphonates are given as a prevention of hypercalcemia, which occurs in $5-10 \%$ of patients ${ }^{50}$. Antimicrobial drugs have been used in the treatment of sarcoidosis mainly in the USA; however, their efficiency has not been proven yet. Only individual patients with skin lesions respond well to the administration of tetracyclines. Bosentan is an endothelin receptor antagonist, that is indicated in patients with pulmonary arterial hypertension associated with pulmonary sarcoidosis. Neurostimulants positively affect fatigue and small fibre neuropathy ${ }^{50}$.

\section{THE TREATMENT OF CARDIAC SARCOIDOSIS}

In general, therapy of cardiac sarcoidosis combines immunosuppressive treatment of inflammation itself together with cardiac-specific pharmacological and nonpharmacological therapeutic approaches.

Early initiation of immunosuppressive therapy prevents progression of ventricular dysfunction and improves clinical outcomes. Despite the lack of placebo-controlled trials, there is a general agreement that corticosteroids should be considered in all patients with cardiac sarcoidosis. The prognosis seems to be better when corticosteroid therapy is initiated even before LV dysfunction develops. The meta-analysis published by Sadek et al. showed that $47 \%$ of patients with high degree AV blocks significantly respond to immunosuppression therapy ${ }^{50}$. In already developed heart failure, the improvement of LV systolic function is seen in patients with only modestly decreased LV EF; however, in cases with advanced LV systolic impairment, no response to therapy is frequent due to the presence of irreversible fibrotic changes within the myocardium ${ }^{51}$. Nevertheless, even in these patients a significant improvement in LV function may be reached by immunosuppressive therapy as shown by Kandolin et 
al. ${ }^{46}$. Thus, early administration of immunosuppressive agents, given in adequate doses, is an important factor of the therapeutic efficacy.

According to current recommendations, oral corticosteroid therapy should be started with the initial dose of $30 \mathrm{mg}(0.5 \mathrm{mg} / \mathrm{kg})$ or $60 \mathrm{mg}(1 \mathrm{mg} / \mathrm{kg})$ of prednisolone daily for the first 4 weeks with subsequent reduction by 5 or $10 \mathrm{mg}$ at intervals of 2 to 4 weeks depending on disease response with final maintenance dose of 5 to $20 \mathrm{mg}$ daily. In patients with diabetes mellites or active infectious diseases, the initial doses are usually lower and given for shorter period. The termination of corticosteroid therapy may be considered after 6-12 months of its administration based on patients' symptoms, clinical findings and results of imaging studies, especially FDG/PET-CT or Galliumscintigraphy ${ }^{51}$. Patients with rapidly progressing cardiac sarcoidosis are treated with initially higher doses or pulse therapy.

In case of relapse, corticosteroids may be administered again as the only treatment, but they are usually combined with other immunosuppressive agent for at least 2 years ${ }^{52}$.

The patients with LV systolic dysfunction and heart failure are treated according to current guidelines ${ }^{53}$. Among antiarrhythmics, betablockers, sotalol and amiodarone are mostly used. However, amiodarone should be administered with caution, because of frequent concomitant lung and liver involvement. Flecainide and propafenone are prohibited because of their proarrhythmic properties. Calcium channels blockers like verapamil are not recommended ${ }^{4}$.

The implantation of permanent pacemaker is indicated in patients with second- or third-degree AV block, with symptoms of bradycardia or with long lasting arrests, even if bradyarrhythmia is reversal with immunosuppression. Immunosuppressive therapy is expected to effect conduction system; however, individual response is unpredictable. The patients with sarcoidosis and high degree AV blocks should receive permanent pacing even without a drug washout period ${ }^{54}$. Importantly, biventricular pacemaker must be considered in all patients indicated to ICD even in the absence of other criteria for resynchronization therapy to prevent further deterioration of LV function due to the unphysiological nature of right ventricular pacing as shown in BLOCK HF study ${ }^{55}$.

Of note, in any case of an indication for permanent pacemaker therapy, a preventive ICD implantation should be considered. In general, ICD is inserted in patients with high risk of sudden death. As a secondary prevention, ICD is clearly indicated in those who survived sudden cardiac arrest or in whom sustained ventricular tachycardia was documented, either spontaneous or induced by electrophysical testing (Class I). Individuals with LVEF $35 \%$ or less despite optimal medical therapy and a period of immunosuppression treatment are also in high risk of ventricular arrhythmias and primary preventive ICD implantation should be considered as a class IIa level of recommendations, if expected survival is greater than 1 year. Importantly, preventive ICD implantation should be considered as a class IIa level of recommendations also in patients with LVEF greater than 35\%, who are symptomatic with syncope of unknown cause, in individuals with LVEF less than 50\% and CMR evidence of extensive myocardial scar and in patients who have other indication for permanent pacing ${ }^{56}$. According to Finish data from the MIDFIN database, risk of sudden cardiac death and malign ventricular arrhythmias is significantly higher also in patients with lone AV block and normal LV systolic function $^{57}$. In this database, $9 \%$ of patients with permanent pacemaker due to high degree AV block and normal LV systolic function experienced sudden cardiac death and $24 \%$ sudden cardiac death or sustained ventricular tachycardia at 5 years follow-up. Furthermore, in patients with AV block and even mildly reduced LV systolic function, the rate of sudden cardiac death was even $14 \%$.

CRT-D is indicated in symptomatic heart failure patients with LVEF lower than 35\% despite optimal drug therapy in whom QRS is prolonged. As already stated above, CRT or CRT-D should be considered in all patients indicated either for implantation of a permanent pacemaker due to bradycardia or to ICD implantation even in the absence of conventional criteria for resynchronization therapy ${ }^{55}$.

The management of ventricular tachyarrhythmias in cardiac sarcoidosis should be modified according to the phase of the disease. Immunosuppression has a major role in patients with evidence of active inflammation as evaluated by FDG-PET/CT. Immunosuppression in combination with antiarrhythmics can reduce ventricular arrhythmia burden in patients with frequent symptomatic ventricular tachycardias and evidence of myocardial inflammation ${ }^{58}$. Catheter ablation may be used in patients with no active inflammation in the myocardium. It is indicated in individuals with ventricular tachycardias uncontrollable by corticosteroids or antiarrhythmics, in those who cannot take antiarrhythmic drugs and in cases of storms of ventricular tachycardias. Due to the nature of the disease, there is usually more than one arrhythmogenic substrate in cardiac sarcoidosis. Therefore, about half of the patients must undergo more than one ablation procedure. The reasons of unsuccessful ablation include tachycardias arising from the ventricular septum, diffuse right ventricular scarring with the presence of several reentry circuits, and tachycardias arising close to the coronary artery or bundle of His (ref. ${ }^{4}$ ).

\section{Heart transplantation}

In the end-stage phase of cardiac sarcoidosis, heart transplant may be considered. According to current guidelines, heart transplantation is indicated in patients with irreversible severe LV systolic dysfunction and heart failure symptoms in NYHA class III-IV, provided they are physically able to undergo transplantation and have adequate social environment. If extracardiac manifestations of sarcoidosis are present, the life prognosis of affected patient should be at least 5 years ${ }^{53}$. Importantly, heart transplantation should always be considered after at least one period of corticosteroid therapy, as this treatment may improve LV systolic function as well as exercise tolerance. 
Patients with cardiac sarcoidosis undergoing heart transplantation have acceptable long-term outcomes, comparable to other patient populations. According to data published by Perkel et al., no significant differences in 5-year freedom from rejection, 5-year survival, 5-year freedom from chronic coronary artery disease and 5-year freedom from nonfatal major adverse cardiac events were seen in 19 patients transplanted for cardiac sarcoidosis as compared to the rest of transplanted cohort counting in total 1069 individuals ${ }^{59}$. Similar results were published recently by Kandolin et al., who demonstrated that outcomes of 12 transplanted patients with cardiac sarcoidosis were like in patients transplanted due to other cardiomyopathies $^{46}$.

Patients with cardiac sarcoidosis who underwent heart transplantation, must be carefully observed as the exacerbation or recurrence of the inflammation may occur. The risk of recurrence seems to be lower in individuals with isolated cardiac sarcoidosis ${ }^{4}$.

Ventricular assist devices are currently indicated in subjects with advanced heart failure during the waiting period for heart transplantation. Patients with cardiac sarcoidosis usually continue to take corticosteroid therapy though there is an increased risk of infection and the benefit of ongoing therapy with steroids has not been clearly documented so far ${ }^{4}$.

\section{CONCLUSIONS}

Cardiac sarcoidosis may be a part of multisystemic disease or present as an isolated heart disorder. High grade AV blocks and ventricular tachyarrhythmias are the most common clinical manifestations, both associated with sudden death. The diagnosis of CS is often challenging and necessitates a multimodality approach with CMR and FDG-PET/CT being the key imaging examinations. Treatment of CS is based on anti-inflammatory therapy, primarily corticosteroid administration, combined with pharmacological as well as non-pharmacological approaches to the treatment of arrhythmias and heart failure, respectively. Despite the advancements in therapy, CS still accounts for substantial mortality. Further research efforts are therefore necessary for a better understanding of its pathogenesis as well as for improving diagnosis and management of CS.

\section{Search strategy and selection criteria}

Our research strategy was aimed at evaluating studies on cardiac manifestations of sarcoidosis. Scientific articles from 1949 to 2021 were searched using the PubMed database. All searches were up to date as of July 2021. The search terms used included "sarcoidosis", "heart", "heart failure", "arrhythmia”, "conduction disorder". Only English language papers were reviewed.

Acknowledgement: The study was supported by Progres Q38/LF1 project.
Author contributions: KK: manuscript writing; TP: manuscript writing and revision; $\mathrm{PK}$ : manuscript revision. Conflict of interest statement: None declared.

\section{REFERENCES}

1. Schupp JC, Freitag-Wolf S, Bargagli, E, Mihailovic-Vucinic V, Rottoli, P, Grubanovic A, Müller A, Jochens A, Tittmann L, Schnerch J, Olivieri C, Fischer A, Jovanovic D, Filipovic S, Videnovic-Ivanovic J, Bresser, P, Jonkers R, O'Reilly K, Ho LP, Gaede KI, Zabel P, Dubaniewicz A, Marshall B, Kieszko R, Milanowski J, Günther A, Weihrich A, Petrek M, Kolek V, Keane MP, O'Beirne S, Donnelly S, Haraldsdottir SO, Jorundsdottir KB, Costabel U, Bonella F, Wallaert B, Grah C, PerošGolubičić T, Luisetti M, Kadija Z, Pabst S, Grohé C, Strausz J, Vašáková M, Sterclova M, Millar A, Homolka J, Slováková A, Kendrick Y, Crawshaw A, Wuyts W, Spencer L, Pfeifer M, Valeyre D, Poletti V, Wirtz H, Prasse1 A, Schreiber S, Krawczak M, Müller-Quernheim J. Phenotypes of organ involvement in sarcoidosis. Eur Respir J 2018;51(1):1700991.

2. Mankad P,Mitchel B, Birnie D, Kron J. Cardiac Sarcoidosis. Curr Cardiol Rep 2019;21(12):152.

3. Grunewald J, Grutters J, Arkema E, Saketkoo L, Moller D, MullerQuernheim J. Sarcoidosis. Nat Rev Dis Primers 2019;5(1):45.

4. Terasaki F, Azuma A, Anzai T, Ishizaka N, Ishida Y, Isobe M, Inomata T, Ishibashi-Ueda H, Eishi Y, Kitakaze M, Kusano K, Sakata Y, Shijubo N, Tsuchida A, Tsutsui H, Nakajima T, Nakatani S, Horii T, Yazaki Y, Yamaguchi E, Yamaguchi T, Ide T, Okamura H, Kato Y, Goya M, Sakakibara M, Soejima K, Nagai T, Nakamura H, Noda T, Hasegawa T, Morita H, Ohe T, Kihara Y, Saito Y, Sugiyama Y, Morimoto SI, Yamashina A; Japanese Circulation Society Joint Working Group. JCS 2016 Guideline on Diagnosis and Treatment of Cardiac Sarcoidosis. Digest Version. Circ J 2019;83(11):2329-88.

5. Arkema EV, Grunewald J, Kullberg S, Eklund A, Askling J. Sarcoidosis incidence and prevalence: a nationwide register-based assessment in Sweden. Eur Respir J 2016;48(6):1690-99.

6. Baughman RP, Field S, Costabel U, Crystal RG, Culver DA, Drent M, Judson MA, Wolff G. Sarcoidosis in America. Analysis Based on Health Care Use. Ann Am Thorac Soc 2016;13(8):1244-52.

7. Yoon HY, Kim H.M, Kim YJ, Song JW. Prevalence and incidence of sarcoidosis in Korea: a nationwide population-based study. Respir Res 2018;19(1):158.

8. Rossides M, Grunewald J, Eklund A, Kullberg S, Di Giuseppe D, Askling J, Arkema EV. Familial aggregation and heritability of sarcoidosis: a Swedish nested case-control study. Eur Respir J 2018;52(2):1800385.

9. Kataoka M, Nakata Y, Hiramatsu J, Kamao T, Tanimoto Y, Kimura G, Tada S, Harada M, Tachibana T, Hiraga Y. Familial occurrence of sarcoidosis in Japan and analysis of genetic influence. The Japanese Journal of Sarcoidosis and Other Granulomatous Disorders 2000;20:21-26.

10. Rybicki BA, lannuzzi MC, Frederick MM, Thompson BW, Rossman MD, Bresnitz EA, Terrin ML, Moller DR, Barnard J, Baughman RP, DePalo L, Hunninghake G, Johns C, Judson MA, Knatterud GL, McLennan G, Newman LS, Rabin DL, Rose C, Teirstein AS, Weinberger SE, Yeager $\mathrm{H}$, Cherniack R; ACCESS Research Group. Familial aggregation of sarcoidosis. A case-control etiologic study of sarcoidosis (ACCESS). Am J Respir Crit Care Med 2001;164(11):2085-91.

11. Shihara M, Ohno S, Ishida T, Ando H, Naruse T, Nose $Y$, Inoko H. Molecular genetic studies of HLA class II alleles in sarcoidosis. Tissue Antigens 1994;43:238-41.

12. Spagnolo $P$, Sato H, Grutters JC, Renzoni EA, Marshall SE, Ruven $\mathrm{HJ}$, Wells AU, Tzouvelekis A, van Moorsel CH, van den Bosch JM, du Bois RM, Welsh KI. Analysis of BTNL2 genetic polymorphisms in British and Dutch patients with sarcoidosis. Tissue Antigens 2007;70(3):2019-227.

13. Foley PJ, McGrath DS, Puscinska E, Petrek M, Kolek V, Drabek J, Lympany PA, Pantelidis P, Welsh KI, Zielinski J, du Bois RM. Human leukocyte antigen-DRB1 position 11 residues are a common protective marker for sarcoidosis. Am J Respir Cell Mol Biol 2001;25(3):27277.

14. Cozier YC, Coogan PF, Govender P, Berman JS, Palmer JR, Rosenberg L. Obesity and weight gain in relation to incidence of sarcoidosis in 
US black women: data from the Black Women's Health Study. Chest 2015;147(4):1086-93.

15. Dumas O, Boggs KM, Cozier YC, Stampfer MJ, Camargo CA Jr. Prospective study of body mass index and risk of sarcoidosis in US women. Eur Respir J 2017;50(4):1701397.

16. Nelson Ct, Kveim reaction in sarcoidosis. Arch Derm Syphilol 1949;60(3):377-89.

17. Siltzbach L. The Kveim test in sarcoidosis: a study of 750 patients. JAMA 1961;178:476-782.

18. Gupta D, Agarwal R, Aggarwal AN, Jindal SK. Molecular evidence for the role of mycobacteria in sarcoidosis: a meta-analysis. Eur Respir $J$ 2007;30(3):508-16.

19. Song Z, Marzilli L, Greenlee BM, Chen ES, Silver RF, Askin FB, Teirstein AS, Zhang Y, Cotter RJ, Moller DR. Mycobacterial catalase-peroxidase is a tissue antigen and target of the adaptive immune response in systemic sarcoidosis. J Exp Med 2005;201(5):755-67.

20. Silverman KJ, Hutchins GM, Bulkley BH. Cardiac sarcoid: a clinicopathologic study of 84 unselected patients with systemic sarcoidosis. Circulation 1978;58:1204-11.

21. Okasha O, Kazmirczak F, Chen KA, Farzaneh-Far A, Shenoy C. Myocardial Involvement in Patients With Histologically Diagnosed Cardiac Sarcoidosis: A Systematic Review and Meta-Analysis of Gross Pathological Images From Autopsy or Cardiac Transplantation Cases. J Am Heart Assoc 2019;8(10):e011253.

22. Ribeiro Neto ML, Jellis $C L$, Joyce $E$, Callahan TD, Hachamovitch R, Culver DA. Update in Cardiac Sarcoidosis. Ann Am Thorac Soc 2019;16(11):1341-50.

23. Kandolin R, Lehtonen J, Kupari M. Cardiac sarcoidosis and giant cell myocarditis as causes of atrioventricular block in young and middleaged adults. Circ Arrhythm Electrophysiol 2011;4(3):303-9.

24. Tung R, Bauer B, Schelbert $H$, Lynch JP 3rd, Auerbach M, Gupta P, Schiepers C, Chan S, Ferris J, Barrio M, Ajijola O, Bradfield J, Shivkumar K. Incidence of abnormal positron emission tomography in patients with unexplained cardiomyopathy and ventricular arrhythmias: The potential role of occult inflammation in arrhythmogenesis. Heart Rhythm 2015;12(12):2488-98.

25. Yoshida A, Ishibashi-Ueda H, Yamada N, Kanzaki H, Hasegawa T, Takahama H, Amaki M, Asakura M, Kitakaze M. Direct comparison of the diagnostic capability of cardiac magnetic resonance and endomyocardial biopsy in patients with heart failure. Eur J Heart Fail 2013;15(2):166-75

26. Raeisi-Giglou P, Rodriguez ER, Blackstone EH, Tan CD, Hsich EM. Verification of Heart Disease: Implications for a New Heart Transplantation Allocation System. JACC Heart Fail 2017;5(12):90413.

27. Birnie DH, Sauer WH, Bogun F, Cooper JM, Culver DA, Duvernoy CS, Judson MA, Kron J, Mehta D, Cosedis Nielsen J, Patel AR, Ohe T, Raatikainen P, Soejima K. HRS expert consensus statement on the diagnosis and management of arrhythmias associated with cardiac sarcoidosis. Heart Rhythm 2014;11(7):1305-23.

28. Banba K, Kusano KF, Nakamura K, Morita H, Ogawa A, Ohtsuka F, Ogo KO, Nishii N, Watanabe A, Nagase S, Sakuragi S, Ohe T. Relationship between arrhythmogenesis and disease activity in cardiac sarcoidosis. Heart Rhythm 2007;4(10):1292-9.

29. Sekhri V, Sanal S, Delorenzo LJ, Aronow WS, Maguire GP. Cardiac sarcoidosis: a comprehensive review. Arch Med Sci 2011;7(4):546-54.

30. Kato $Y$, Morimoto S. Diagnosis and treatment of cardiac sarcoidosis. The Japanese Journal of Sarcoidosis and Other Granulomatous Disorders 2008;28:15-24.

31. Roberts WC, MCAllister HA Jr, Ferrans VJ. Sarcoidosis of the heart. A clinicopathologic study of 35 necropsy patients (group 1) and review of 78 previously described necropsy patients (group 11). Am J Med 1977;63(1):86-108.

32. Pizarro C, Kluenker F, Hammerstingl C, Skowasch D. Diagnostic value of speckle-tracking echocardiography in confirmed cardiac sarcoidosis. Clin Res Cardiol 2016;105(10):884-6.

33. Smedema JP, Snoep G, van Kroonenburgh MP, van Geuns RJ, Dassen WR, Gorgels AP, Crijns HJ. Evaluation of the accuracy of gadoliniumenhanced cardiovascular magnetic resonance in the diagnosis of cardiac sarcoidosis. J Am Coll Cardiol 2005;45(10):1683-90.

34. Coleman GC, Shaw PW, Balfour PC Jr, Gonzalez JA, Kramer CM Patel AR, Salerno M. Prognostic Value of Myocardial Scarring on CMR in Patients With Cardiac Sarcoidosis. JACC Cardiovasc Imaging 2017;10(4):411-20
35. Writing group; Document reading group; EACVI Reviewers: This document was reviewed by members of the EACVI Scientific Documents Committee for 2014-2016 and 2016-2018. A joint procedural position statement on imaging in cardiac sarcoidosis: from the Cardiovascular and Inflammation \& Infection Committees of the European Association of Nuclear Medicine, the European Association of Cardiovascular Imaging, and the American Society of Nuclear Cardiology. Eur Heart J Cardiovasc Imaging 2017;18(10):1073-89.

36. Kim SJ, Pak K, Kim K. Diagnostic performance of F-18 FDG PET for detection of cardiac sarcoidosis; a systematic review and metaanalysis. J Nucl Cardiol 2020;27(6):2103-15.

37. Wicks EC, Menezes LJ, Barnes A, Mohiddin SA, Sekhri N, Porter JC, Booth HL, Garrett E, Patel RS, Pavlou M, Groves AM, Elliott PM. Diagnostic accuracy and prognostic value of simultaneous hybrid 18F-fluorodeoxyglucose positron emission tomography/magnetic resonance imaging in cardiac sarcoidosis. Eur Heart J Cardiovasc Imaging 2018;19(7):757-67.

38. Sperry BW, Tamarappoo BK, Oldan JD, Javed O, Culver DA, Brunken R, Cerqueira MD, Hachamovitch R. Prognostic Impact of Extent, Severity, and Heterogeneity of Abnormalities on 18F-FDG PET Scans for Suspected Cardiac Sarcoidosis. JACC Cardiovasc Imaging 2018;11(2 Pt 2):336-45.

39. Vaidya VR, Abudan AA, Vasudevan K, Shantha G, Cooper LT, Kapa S, Noseworthy PA, Cha YM, Asirvatham SJ, Deshmukh AJ. The efficacy and safety of electroanatomic mapping-guided endomyocardial biopsy: a systematic review. J Interv Card Electrophysiol 2018;53(1):6371.

40. Kiko T, Yoshihisa A, Kanno Y, Yokokawa T, Abe S, Miyata-Tatsumi M, Misaka T, Oikawa M, Kobayashi A, Ishida T, Takeishi Y. A Multiple Biomarker Approach in Patients with Cardiac Sarcoidosis. Int Heart J 2018;59(5):996-1001.

41. Martusewicz-Boros MM, Boros PW, Wiatr E, Zych J, PiotrowskaKownacka D, Roszkowski-Śliż K. Prevalence of cardiac sarcoidosis in white population: a case-control study: Proposal for a novel risk index based on commonly available tests. Medicine (Baltimore) 2016;95(32):4518.

42. Mehta D, Mori N, Goldbarg SH, Lubitz S, Wisnivesky JP, Teirstein A. Primary prevention of sudden cardiac death in silent cardiac sarcoidosis: role of programmed ventricular stimulation. Circ Arrhythm Electrophysiol 2011;4(1):43-8.

43. Birnie DH, Sauer WH, Bogun F, Cooper JM, Culver DA, Duvernoy CS, Judson MA, Kron J, Mehta D, Cosedis Nielsen J, Patel AR, Ohe T, Raatikainen P, Soejima K. HRS expert consensus statement on the diagnosis and management of arrhythmias associated with cardiac sarcoidosis. Heart Rhythm 2014;11(7):1305-23.

44: Sugie T, Hashimoto N, Iwai K. Clinical and autopsy studies on prognosis of sarcoidosis. Nihon Rinsho 1994;52(6):1567-70.

45. Yazaki Y, Isobe M, Hiroe M, Morimoto S, Hiramitsu S, Nakano T, Izumi T, Sekiguchi M; Central Japan Heart Study Group. Prognostic determinants of long-term survival in Japanese patients with cardiac sarcoidosis treated with prednisone. Am J Cardiol 2001;88(9):100610.

46. Kandolin R, Lehtonen J, Airaksinen J, Vihinen T, Miettinen $\mathrm{H}$, Ylitalo $\mathrm{K}$, Kaikkonen K, Tuohinen S, Haataja P, Kerola T, Kokkonen J, Pelkonen M, Pietilä-Effati P, Utrianen S, Kupari M. Cardiac sarcoidosis: epidemiology, characteristics, and outcome over 25 years in a nationwide study. Circulation 2015;131(7):624-32.

47. Joyce E, Ninaber MK, Katsanos S, Debonnaire P, Kamperidis V, Bax Jנ, Taube C, Delgado V, Ajmone Marsan N. Subclinical left ventricular dysfunction by echocardiographic speckle-tracking strain analysis relates to outcome in sarcoidosis. Eur J Heart Fail 2015;17(1):51-62.

48. Blankstein R, Osborne M, Naya M, Waller A, Kim CK, Murthy VL, Kazemian P, Kwong RY, Tokuda M, Skali H, Padera R, Hainer J, Stevenson WG, Dorbala S, Di Carli MF. Cardiac positron emission tomography enhances prognostic assessments of patients with suspected cardiac sarcoidosis. J Am Coll Cardiol 2014;63(4):329-36.

49. Grunewald J, Grutters JC, Arkema EV, Saketkoo LA, Moller DR, MüllerQuernheim J. Sarcoidosis. Nat Rev Dis Primers 2019;5(1):45.

50. Sadek MM, Yung D, Birnie DH, Beanlands RS, Nery PB. Corticosteroid therapy for cardiac sarcoidosis: a systematic review. Can J Cardiol 2013;29(9):1034-41.

51. The Japan Society of Sarcoidosis and Other Granulomatous Disorders, the Japanese Respiratory Society, the Japanese College of Cardiology, et al. Sarcoidosis Treatment Guideline Preparation 
Committee. Views on the treatment of sarcoidosis - 2003. The Japanese Journal of Sarcoidosis and Other Granulomatous Disorders 2003;23:105-14.

52. James WE, Baughman R. Treatment of sarcoidosis: grading the evidence. Expert Rev Clin Pharmacol 2018;11(7):677-87.

53. Ponikowski P, Voors AA, Anker SD, Bueno H, Cleland JGF, Coats AJS, Falk V, González-Juanatey JR, Harjola VP, Jankowska EA, Jessup M, Linde C, Nihoyannopoulos P, Parissis JT, Pieske B, Riley JP, Rosano GMC, Ruilope LM, Ruschitzka F, Rutten FH, van der Meer P; ESC Scientific Document Group. 2016 ESC Guidelines for the diagnosis and treatment of acute and chronic heart failure: The Task Force for the diagnosis and treatment of acute and chronic heart failure of the European Society of Cardiology (ESC) Developed with the specia contribution of the Heart Failure Association (HFA) of the ESC. Eur Heart J 2016;37(27):2129-200.

54. Kusumoto FM, Schoenfeld $\mathrm{MH}$, Barrett $\mathrm{C}$, Edgerton JR, Ellenbogen KA, Gold MR, Goldschlager NF, Hamilton RM, Joglar JA, Kim RJ, Lee R, Marine JE, McLeod CJ, Oken KR, Patton KK, Pellegrini CN, Selzman KA, Thompson A, Varosy PD. 2018 ACC/AHA/HRS Guideline on the Evaluation and Management of Patients With Bradycardia and Cardiac Conduction Delay: A Report of the American College of Cardiology/American Heart Association Task Force on Clinica Practice Guidelines and the Heart Rhythm Society. Circulation 2019;140(8):e382-e482.
55. Curtis AB, Worley SJ, Adamson PB, Chung ES, Niazi I, Sherfesee L, Shinn T, Sutton MS; Biventricular versus Right Ventricular Pacing in Heart Failure Patients with Atrioventricular Block (BLOCK HF) Tria Investigators. Biventricular pacing for atrioventricular block and systolic dysfunction. N Engl J Med 2013;368(17):1585-93.

56. Kazmirczak F, Chen KA, Adabag S, von Wald L, Roukoz H, Benditt DG, Okasha O, Farzaneh-Far A, Markowitz J, Nijjar PS, Velangi PS, Bhargava M, Perlman D, Duval S, Akçakaya M, Shenoy C. Assessment of the 2017 AHA/ACC/HRS Guideline Recommendations for Implantable Cardioverter-Defibrillator Implantation in Cardiac Sarcoidosis. Circ Arrhythm Electrophysiol 2019;12(9):e007488.

57. Nordenswan HK, Lehtonen J, Ekström K, Kandolin R, Simonen $P$, Mäyränpää M, Vihinen T, Miettinen $H$, Kaikkonen $K$, Haataja $P$, Kerola T, Rissanen TT, Kokkonen J, Alatalo A, Pietilä-Effati P, Utriainen S, Kupari M. Outcome of Cardiac Sarcoidosis Presenting With High-Grade Atrioventricular Block. Circ Arrhythm Electrophysiol 2018;11(8):e006145.

58. Naruse $Y$, Sekiguchi $Y$, Nogami A, Okada $H$, Yamauchi $Y$, Machino T, Kuroki K, Ito Y, Yamasaki H, Igarashi M, Tada H, Nitta J, Xu D, Sato A, Aonuma K. Systematic treatment approach to ventricular tachycardia in cardiac sarcoidosis. Circ Arrhythm Electrophysiol 2014;7(3):407-13.

59. Perkel D, Czer LS, Morrissey RP, Ruzza A, Rafiei M, Awad M, Patel J, Kobashigawa JA. Heart transplantation for end-stage heart failure due to cardiac sarcoidosis. Transplant Proc 2013;45(6):2384-6. 